\title{
ON NORMALITY OF BLASCHKE QUOTIENTS
}

\author{
FLAVIA COLONNA
}

(Communicated by Irwin Kra)

\begin{abstract}
In this paper we give conditions on the zeros and poles of a Blaschke quotient in order to obtain a normal meromorphic function, extending the condition for normality given by Cima and Colwell to the case of zeros and poles forming interpolating sequences and of bounded multiplicities. It is also shown that a similar characterization does not apply in the case of unbounded multiplicities.
\end{abstract}

1. Normal functions: basic definitions and results. Let $\chi$ be the chordal metric on $\hat{\mathbf{C}}$, the extended plane, normalized so that $\chi(0, \infty)=1$.

If $f$ is a meromorphic function defined on $\Delta$, the open unit disk, then the spherical derivative of $f$ is the nonnegative function $\sigma_{f}$ which assigns to every point $\varsigma \in \Delta$ the value

$$
\sigma_{f}(\varsigma)=\lim _{z \rightarrow \varsigma} \frac{\chi[f(z), f(\varsigma)]}{|z-\varsigma|}
$$

Set

$$
\alpha_{f}=\sup _{z \in \Delta}\left(1-|z|^{2}\right) \sigma_{f}(z)=\sup _{z \in \Delta} \frac{\left(1-|z|^{2}\right)\left|f^{\prime}(z)\right|}{1+|f(z)|^{2}} .
$$

DEFinition. The function $f$ is called normal provided that $\alpha_{f}<+\infty$. The number $\alpha_{f}$ is termed the normality constant of $f$.

The notion of a normal meromorphic function was introduced by Lehto and Virtanen in [6]. The justification for the terminology is based on an equivalence given by the following result.

THEOREM 1. A meromorphic function with domain $\Delta$ is normal if and only if the collection

$$
\mathcal{F}_{f}=\{f \circ S \mid S \text { a conformal automorphism of } \Delta\}
$$

is a normal family; namely, every sequence in $\mathcal{F}_{f}$ admits a subsequence converging on compact subsets, possibly with limit $\infty$.

The Theorem of Arzelà-Ascoli asserts that a family of continuous functions on $\Delta$ with images in a compact metric space is normal if and only if it is equicontinuous on each compact subset of $\Delta$ (cf. $[1$, p. 222]). In the special case of $\hat{\mathbf{C}}$ endowed with the chordal metric $\chi$, a meromorphic function $f$ on $\Delta$ is normal if and only if the family $\mathcal{F}_{f}$ is equicontinuous on compact subsets of $\Delta$.

Received by the editors October 8, 1986.

1980 Mathematics Subject Classification (1985 Revision). Primary 30D45; Secondary 30D50.

The results in this paper are contained in the author's Ph.D. dissertation written at the University of Maryland in 1985. The author wishes to express thanks and gratitude to her advisor, Professor Maurice Heins, for his time, guidance, and great patience. 
We recall that the hyperbolic distance $\rho\left(z_{1}, z_{2}\right)$ between two points $z_{1}$ and $z_{2}$ in $\Delta$ is defined by

$$
\rho\left(z_{1}, z_{2}\right)=\frac{1}{2} \log \left(\frac{1+\left|L_{z_{1}}\left(z_{2}\right)\right|}{1-\left|L_{z_{1}}\left(z_{2}\right)\right|}\right)
$$

where $L_{a}(z)=(a-z) /(1-\bar{a} z)$, for $a, z \in \Delta$.

By its invariance under composition with a conformal automorphism of $\Delta$, the equicontinuity on compact subsets of $\Delta$ of the family $\mathcal{F}_{f}$ is equivalent to the uniform continuity of $f$ as a map of the metric space $(\Delta, \rho)$ to $(\hat{\mathbf{C}}, \chi)$. As a consequence, we have

THEOREM 2. Let $w_{1} \neq w_{2} \in \hat{\mathbf{C}}$, and let $f$ be a normal meromorphic function taking the values $w_{1}$ and $w_{2}$. Assume that $f^{-1}\left(\left\{w_{1}\right\}\right)$ and $f^{-1}\left(\left\{w_{2}\right\}\right)$ form two infinite sets:

$$
f^{-1}\left(\left\{w_{1}\right\}\right)=\left\{z_{k} \mid k \in \mathbf{N}\right\} \quad \text { and } f^{-1}\left(\left\{w_{2}\right\}\right)=\left\{\zeta_{k} \mid k \in \mathbf{N}\right\} .
$$

Then there exists a positive constant $c$ such that $\rho\left(z_{k}, \zeta_{j}\right) \geq c$ for all $k, j \in \mathbf{N}$. In particular, if $f$ has an infinity of zeros and an infinity of poles, the $\rho$-distance of a zero from a pole cannot become arbitrarily small.

2. Normality of Blaschke quotients. By a Blaschke quotient we mean the quotient of two Blaschke products with no zeros in common. We shall use the notation $B_{\left(z_{n}\right)}$ for the Blaschke product with zeros $\left(z_{n}\right)$ and $Q_{\left(z_{n} ; w_{n}\right)}$ for the Blaschke quotient $B_{\left(z_{n}\right)} / B_{\left(w_{n}\right)}$.

If $\left(z_{n}\right)$ and $\left(w_{n}\right)$ are the zeros and the poles of a normal function $f$, then $\inf _{m, n \in \mathbb{N}} \rho\left(z_{m}, w_{n}\right)$ cannot be zero, by Theorem 2 .

Our aim is to obtain some kind of converse in the special case of a Blaschke quotient. It is handy to introduce an alternative metric on $\Delta$ closely related to $\rho$. For $z, w \in \Delta$ define $\Psi(z, w)=|(z-w) /(1-\bar{z} w)|$. The function $\Psi$ is a metric endowing $\Delta$ with the standard topology. It is called the pseudohyperbolic metric of $\Delta$ (cf. [4, p. 2]) and is related to the hyperbolic distance by

$$
\rho(z, w)=\frac{1}{2} \log \frac{1+\Psi(z, w)}{1-\Psi(z, w)}, \quad z, w \in \Delta .
$$

This formula shows that the two metrics are locally equivalent.

DEFINITION. A sequence $\left(z_{n}\right)$ of points of $\Delta$ is called interpolating in $\Delta$ if for every bounded sequence $\left(w_{n}\right)$ there exists a bounded analytic function $f$ such that $f\left(z_{n}\right)=w_{n}, n \in \mathbf{N}$.

Carleson characterized interpolating sequences in terms of the pseudohyperbolic metric by the following theorem.

THEOREM 3. A sequence $\left(z_{n}\right)$ is interpolating if and only if there exists a positive constant $\delta$ such that

$$
\prod_{k \neq n} \Psi\left(z_{k}, z_{n}\right) \geq \delta \quad \text { for all } n \in \mathbf{N}
$$

(Cf. [4, pp. 284-294] or [2, pp. 921-930] for Carleson's original proof; [4, pp. 308313] for an elementary proof due to J. P. Earl.) 
In [3] Cima and Colwell characterized the normality of a Blaschke quotient whose zeros and poles are simple and form interpolating sequences:

THEOREM 4. Let $\left(z_{n}\right)$ and $\left(w_{n}\right)$ be interpolating sequences in $\Delta$. Then $Q_{\left(z_{n} ; w_{n}\right)}$ is normal if and only if the sequence whose elements are $z_{n}$ and $w_{n}, n \in \mathbf{N}$, is interpolating.

Theorem 4 applies only to Blaschke quotients with simple zeros and poles. We shall establish a similar necessary and sufficient condition for a Blaschke quotient for which there is a bound on the multiplicities of the zeros and poles.

LEMMA 1. Let $Q=Q_{\left(z_{n} ; w_{n}\right)}$ be nonnormal. Then there exists a sequence $\left(a_{k}\right)_{k \in \mathbf{N}}$ in $\Delta$ such that $\left|a_{k}\right| \rightarrow 1, B_{\left(z_{n}\right)}\left(a_{k}\right) \rightarrow 0$, and $B_{\left(w_{n}\right)}\left(a_{k}\right) \rightarrow 0$.

PrOOF. Since $Q$ is not normal, there exists a sequence $\left(a_{k}\right)_{k \in \mathbf{N}}$ in $\Delta$ such that $\left|a_{k}\right| \rightarrow 1$ and

$$
\lim _{k \rightarrow \infty}\left(1-\left|a_{k}\right|^{2}\right) \sigma_{Q}\left(a_{k}\right)=+\infty
$$

Since $B_{\left(z_{n}\right)}$ and $B_{\left(w_{n}\right)}$ have modulus less than 1 in $\Delta$, we have $\left(1-|z|^{2}\right)\left|B_{\left(z_{n}\right)}^{\prime}(z)\right| \leq$ 1 and $\left(1-|z|^{2}\right)\left|B_{\left(w_{n}\right)}^{\prime}(z)\right| \leq 1$, for $|z|<1$. Thus

$$
\begin{aligned}
(1- & \left.\left|a_{k}\right|^{2}\right) \sigma_{Q}\left(a_{k}\right) \\
& =\frac{\left(1-\left|a_{k}\right|^{2}\right)\left|B_{\left(z_{n}\right)}^{\prime}\left(a_{k}\right) B_{\left(w_{n}\right)}\left(a_{k}\right)-B_{\left(z_{n}\right)}\left(a_{k}\right) B_{\left(w_{n}\right)}^{\prime}\left(a_{k}\right)\right|}{\left|B_{\left(z_{n}\right)}\left(a_{k}\right)\right|^{2}+\left|B_{\left(w_{n}\right)}\left(a_{k}\right)\right|^{2}} \\
& \leq \frac{\left(1-\left|a_{k}\right|^{2}\right)\left|B_{\left(z_{n}\right)}^{\prime}\left(a_{k}\right)\right|}{\left|B_{\left(z_{n}\right)}\left(a_{k}\right)\right|^{2}+\left|B_{\left(w_{n}\right)}\left(a_{k}\right)\right|^{2}}+\frac{\left(1-\left|a_{k}\right|^{2}\right)\left|B_{\left(w_{n}\right)}^{\prime}\left(a_{k}\right)\right|}{\left|B_{\left(z_{n}\right)}\left(a_{k}\right)\right|^{2}+\left|B_{\left(w_{n}\right)}\left(a_{k}\right)\right|^{2}} \\
& \leq \frac{2}{\left|B_{\left(z_{n}\right)}\left(a_{k}\right)\right|^{2}+\left|B_{\left(w_{n}\right)}\left(a_{k}\right)\right|^{2}} .
\end{aligned}
$$

From (2) and (3) we conclude that $\left|B_{\left(z_{n}\right)}\left(a_{k}\right)\right| \rightarrow 0$ and $\left|B_{\left(w_{n}\right)}\left(a_{k}\right)\right| \rightarrow 0$.

If we add the assumption that the sequences $\left(z_{n}\right)$ and $\left(w_{n}\right)$ are interpolating and that their multiplicities are bounded, then Lemma 1 admits a converse, Lemma 2. The proof we give uses the following property of interpolating sequences, proved by Cima and Colwell (cf. [3, p. 796]).

THEOREM 5. If $\left(z_{n}\right)$ is interpolating, then for each $\varepsilon>0$ there is $\eta>0$ such that $\left|B_{\left(z_{n}\right)}(z)\right|>\eta$ whenever $\Psi\left(z, z_{n}\right) \geq \varepsilon, n \in \mathbf{N}$.

LEMMA 2. Let $\left(z_{n}\right)$ and $\left(w_{n}\right)$ be interpolating in $\Delta$, and let $Q=B_{\left(z_{n}\right)}^{*} / B_{\left(w_{n}\right)}^{*}$ denote a Blaschke quotient with zeros $\left(z_{n}\right)$ and poles $\left(w_{n}\right)$ with a bound on their multiplicities. Let $\left(a_{k}\right)_{k \in \mathbf{N}}$ be a sequence in $\Delta$ such that $\left|a_{k}\right| \rightarrow 1, B_{\left(z_{n}\right)}^{*}\left(a_{k}\right) \rightarrow 0$, and $B_{\left(w_{n}\right)}^{*}\left(a_{k}\right) \rightarrow 0$. Then $Q$ is not normal.

PROOF. By Theorem 5 there exists a subsequence $\left(a_{n(k)}\right)$ such that

$$
\lim _{k} \Psi\left(a_{n(k)}, z_{n(k)}\right)=0
$$

and similarly, the latter sequence admits a subsequence $\left(a_{m(k)}\right)$ such that

$$
\lim _{k} \Psi\left(a_{m(k)}, w_{m(k)}\right)=0 .
$$

Then $\lim _{k} \Psi\left(z_{m(k)}, w_{m(k)}\right)=0$ by the triangle inequality. Thus by (1) we have $\rho\left(z_{m(k)}, w_{m(k)}\right)$ tends to zero. Hence $Q$ is not normal. 
THEOREM 6. Let $Q$ be a Blaschke quotient with distinct zeros $\left(z_{n}\right)$ and poles $\left(w_{n}\right)$ forming two interpolating sequences in $\Delta$, not necessarily simple. Suppose that there exists a positive integer $M$ such that the multiplicities $\nu_{n}$ and $\mu_{n}$ of $Q$ at $z_{n}$ and $w_{n}$ respectively are bounded above by $M$ for all $n \in \mathrm{N}$. Then $Q$ is normal if and only if the sequence formed by the zeros and poles of $Q$ is interpolating.

ProOF. Denote by $B_{\left(z_{n}\right)}^{*}, B_{\left(w_{n}\right)}^{*}$ the two Blaschke products defining $Q$ and by $B_{\left(z_{n}\right)}, B_{\left(w_{n}\right)}$ the Blaschke products having the same zeros as $B_{\left(z_{n}\right)}^{*}$ and $B_{\left(w_{n}\right)}^{*}$, respectively, all simple. Since every zero of $B_{\left(z_{n}\right)}^{*}$ and $B_{\left(\hat{w}_{n}\right)}^{*}$ has multiplicity no greater than $M$ we have

$$
\left|B_{\left(z_{n}\right)}\right|^{M} \leq\left|B_{\left(z_{n}\right)}^{*}\right| \leq\left|B_{\left(z_{n}\right)}\right| \text { and }\left|B_{\left(w_{n}\right)}\right|^{M} \leq\left|B_{\left(w_{n}\right)}^{*}\right| \leq\left|B_{\left(w_{n}\right)}\right| .
$$

Thus by Lemmas 1 and 2 and (4), the Blaschke quotient $Q$ is not normal if and only if there exists a sequence $\left(a_{k}\right)_{k \in \mathbf{N}}$ in $\Delta$ with $\lim \left|a_{k}\right|=1, \lim \left|B_{\left(z_{n}\right)}\left(a_{k}\right)\right|=$ $\lim \left|B_{\left(w_{n}\right)}\left(a_{k}\right)\right|=0$. But this statement is equivalent to saying that the Blaschke quotient $Q_{1}=B_{\left(z_{n}\right)} / B_{\left(w_{n}\right)}$ is not normal. By Theorem $4, Q_{1}$ is normal if and only if its zeros and poles form an interpolating sequence.

It is natural to ask whether Theorem 6 can be extended to a Blaschke quotient with no restriction at all on the multiplicities of its zeros and/or poles. The answer is "no in general" as we shall see in Theorem 7.

Suppose $B_{\left(z_{n}\right)}^{*}$ is a Blaschke product with zeros $\left(z_{n}\right)$, an interpolating sequence in $\Delta$, and let $\nu_{n}$ denote the multiplicity at $z_{n}$, with $\lim _{n \rightarrow \infty} \nu_{n}=+\infty$. For $z \in \Delta$ the inequality $\left|B_{\left(z_{n}\right)}^{*}(z)\right| \leq\left|B_{\left(z_{n}\right)}(z)\right|$ holds, so that if $\left(a_{k}\right)_{k \in \mathbf{N}}$ is a sequence in $\Delta$ satisfying the condition $\left|B_{\left(z_{n}\right)}\left(a_{k}\right)\right| \rightarrow 0$ then $\left|B_{\left(z_{n}\right)}^{*}\left(a_{k}\right)\right| \rightarrow 0$. However the converse is not true in general as we see in the following

LEMMA 3. There exists a Blaschke product $B^{*}$ with zeros $\left(z_{n}\right)$ and a sequence $\left(a_{k}\right)$ such that

$$
\begin{gathered}
0<\left|a_{k}\right|<1, \quad \lim _{k \rightarrow \infty}\left|a_{k}\right|=1, \\
\lim _{k \rightarrow \infty}\left|B^{*}\left(a_{k}\right)\right|=0, \quad \text { and } \quad \lim _{k \rightarrow \infty}\left|B_{\left(z_{n}\right)}\left(a_{k}\right)\right|>0 .
\end{gathered}
$$

ProOF. Let $0<\lambda<+\infty$ and set $\phi_{\lambda}=\operatorname{inv}(T) \circ R_{\lambda} \circ T$, where $T(z)=$ $(1+z) /(1-z)$ and $R_{\lambda}(z)=\lambda z$. The family $\left\{\phi_{\lambda}: 0<\lambda<+\infty\right\}$ forms a 1 parameter abelian group under composition: $\phi_{\lambda} \circ \phi_{\mu}=\phi_{\lambda \mu}$ for $\lambda, \mu>0$. In particular, $\phi_{\lambda^{n}}=\phi_{\lambda}^{n}$ for $n \in \mathbf{N}$. Furthermore $\phi_{\lambda^{-1}}(z)=-\phi_{\lambda}(-z)$.

Fix $\lambda>1$, and let $\tau=\phi_{\lambda}$ and $\tau_{n}=\phi_{\lambda^{n}}, n \in \mathbf{N}$. Then $\tau$ is a hyperbolic conformal automorphism of $\Delta$ with fixed points -1 and 1 , with 1 attractive. Denote by $\tau_{-n}$, $n$ positive, the $n$th iterate of $\operatorname{inv}(\tau)$.

We construct a sequence $\left(z_{n}\right)_{n \in \mathbf{Z}}$ as an orbit of $\left(\tau_{n}\right)_{n \in \mathbf{Z}}$ by setting $z_{n}=\tau_{n}(0)=$ $\left(\lambda^{n}-1\right) /\left(\lambda^{n}+1\right)$. Then $\left(z_{n}\right)$ is strictly increasing, $-1<z_{n}<1, \tau\left(z_{n}\right)=z_{n+1}$, $\inf \left(z_{n}\right)=-1$, and $\sup \left(z_{n}\right)=1$. Moreover, the Blaschke condition $\sum_{n \in \mathbf{Z}}\left(1-\left|z_{n}\right|\right)<$ $+\infty$ is satisfied:

Thus

$$
1-\left|z_{n}\right|=1-\frac{\lambda^{n}-1}{\lambda^{n}+1}=\frac{2}{\lambda^{n}+1}=1-\left|z_{-n}\right| \text {. }
$$

$$
\sum_{n \in \mathbf{Z}}|n|\left(1-\left|z_{n}\right|\right)=4 \sum_{n=1}^{\infty} \frac{n}{\lambda^{n}+1}<4 \sum_{n=1}^{\infty} n \lambda^{-n}<+\infty
$$

since $\lambda>1$. 
We now construct the normalized convergent Blaschke product $B^{*}$ having each $z_{n}$ as a zero of multiplicity $|n|$ and no others. We observe that both $B=B_{\left(z_{n}\right)}$ and $B^{*}$ take on real values on the interval $(-1,1)$ and that $B \circ \tau$ and $B$ are negatives of one another. In fact, $L_{z_{n+1}} \circ \tau=L_{z_{n}}$, since $\tau\left(z_{n}\right)=z_{n+1}$ and both functions take on the same values at -1 and 1 . Thus

$$
B \circ \tau=\prod_{n \in \mathbf{Z}} \operatorname{sg}\left(z_{n+1}\right) L_{z_{n+1}} \circ \tau=-\prod_{n \in \mathbf{Z}} \operatorname{sg}\left(z_{n}\right) L_{z_{n}}=-B
$$

where

$$
\operatorname{sg}\left(z_{n}\right)=\left\{\begin{aligned}
1 & \text { for } n>0 \\
-1 & \text { otherwise }
\end{aligned}\right.
$$

So $\left|B \circ \tau_{k}\right|=|B|$ for all $k$.

Next we construct a sequence $\left(a_{k}\right)_{k \in \mathbf{Z}}$ satisfying (5). Let $a$ be a point of the interval $[0, \tau(0)]$ where the maximum of $B$ is attained. Let $m=B(a)$. Then $m \in(0,1)$ because $B^{\prime}(0)=\prod_{n=1}^{\infty} z_{n}^{2}>0$. Since $|B \circ \tau|=|B|$ the value $m$ is attained by $|B|$ at each point of the orbit $\left\{\tau_{k}(a)\right\}$. Set $a_{k}=\tau_{k}(a), k \in \mathbf{Z}$. Then $\lim _{k \rightarrow \infty}\left|B\left(a_{k}\right)\right|=m>0$.

Next we prove that $B^{*}$ has zero as radial limit at the point 1 . Fix $r \in(m, 1)$. Let $n$ be a positive integer. The function $z \mapsto \prod_{k<n} L_{z_{k}}(z)$ is analytic at 1 and takes either the value 1 or -1 at 1 . So for all $z \in(0,1)$ sufficiently close to 1 , we have $\prod_{k<n}\left|L_{z_{k}}(z)\right|>r$. Hence for all such $z$ we have

$$
\left|B^{*}(z)\right| \leq \prod_{k=n}^{\infty}\left|L_{z_{k}}(z)\right|^{n}=\left(|B(z)| / \prod_{k<n}\left|L_{z_{k}}(z)\right|\right)^{n}<(m / r)^{n} .
$$

By letting $n \rightarrow+\infty$, we conclude that $B^{*}$ has radial limit zero at 1 . In particular, $\lim _{k \rightarrow+\infty}\left|B^{*}\left(a_{k}\right)\right|=0$ and the assertion of the preceding paragraph follows.

LEMMA 4. The sequences $\left(z_{n}\right)$ and $\left(a_{k}\right)$ of Lemma 3 are interpolating.

PROOF. In the proof of Lemma 3, we saw that the Blaschke condition

$$
\sum_{n \in \mathbf{Z}}\left(1-\left|z_{n}\right|\right)<+\infty
$$

is satisfied. Therefore $\delta=\prod_{n \neq 0}\left|z_{n}\right|$ is not zero. We observe that

$$
\left(z_{n}-z_{k}\right) /\left(1-z_{n} z_{k}\right)=L_{z_{n}}\left(z_{k}\right)=L_{z_{n}}\left[\tau_{k}(0)\right]=L_{z_{n-k}}(0)=z_{n-k} .
$$

Thus

$$
\prod_{n \neq k} \Psi\left(z_{n}, z_{k}\right)=\prod_{n \neq k}\left|z_{n-k}\right|=\delta
$$

Hence $\left(z_{n}\right)$ is interpolating by Theorem 3. Let $\sigma$ denote the Möbius transformation with fixed points $-1,1$ sending 0 to $a: \sigma(z)=(a+z) /(1+a z)$. By the definitions of $\tau_{n}$ and $z_{n}$, we have $\tau_{n}(z)=\left(z_{n}+z\right) /\left(1+z_{n} z\right)$. So $\sigma\left(z_{n}\right)=\left(a+z_{n}\right) /\left(1+a z_{n}\right)=$ $\tau_{n}(a)=a_{n}$. Since $\sigma$ is a conformal autormorphism of $\Delta$, by the invariance of the pseudohyperbolic metric under conformal automorphisms of $\Delta$, we have

$$
\Psi\left(a_{n}, a_{k}\right)=\Psi\left[\sigma\left(z_{n}\right), \sigma\left(z_{k}\right)\right]=\Psi\left(z_{n}, z_{k}\right) .
$$

By (6), (7) and Theorem 3 we conclude that $\left(a_{k}\right)$ is an interpolating sequence. 
With $B^{*}, B_{\left(z_{n}\right)}$ and $\left(a_{k}\right)_{k \in \mathbf{Z}}$ as in Lemma 3, we now show

THEOREM 7. There exists a Blaschke product $B$ such that $Q=B^{*} / B$ is not normal but $B_{\left(z_{n}\right)} / B$ is normal.

Before giving the proof of Theorem 7 we observe that the Carleson criterion of Theorem 3 can be formulated in terms of the derivative of the Blaschke product $B$ associated with a sequence as follows:

LEMMA 5. A sequence $\left(z_{n}\right)$ is interpolating in $\Delta$ if and only if there exists $\delta>0$ such that for all $n \in \mathbf{N}$ the quantity $\left(1-\left|z_{n}\right|^{2}\right)\left|B^{\prime}\left(z_{n}\right)\right|$ is bounded below by $\delta$. Here $B$ is the Blaschke product associated with $\left(z_{n}\right)$.

PrOOF. To show this equivalence fix $n$ and define $B_{n}=B / L_{z_{n}}$. Then

$$
\left|B^{\prime}\left(z_{n}\right)\right|=\left|L_{z_{n}}^{\prime}\left(z_{n}\right) B_{n}\left(z_{n}\right)\right|=\frac{1}{1-\left|z_{n}\right|^{2}} \prod_{k \neq n} \Psi\left(z_{k}, z_{n}\right) .
$$

Thus $\left(1-\left|z_{n}\right|^{2}\right)\left|B^{\prime}\left(z_{n}\right)\right|=\prod_{k \neq n} \Psi\left(z_{k}, z_{n}\right)$ and the assertion follows from Theorem 3.

PROOF OF THEOREM 7 . Since $\left(z_{n}\right)$ and $\left(a_{k}\right)$ are interpolating sequences there exists an $\varepsilon>0$ such that $\prod_{j \neq n} \Psi\left(z_{n}, z_{j}\right) \geq \varepsilon, \prod_{j \neq n} \Psi\left(a_{n}, a_{j}\right) \geq \varepsilon$ for all $n \in \mathbf{Z}$. Furthermore $\left|B_{\left(z_{n}\right)}\left(a_{k}\right)\right|=\prod_{n} \Psi\left(z_{n}, a_{k}\right)$ is bounded below by a positive constant $c$ (cf. (5)). Let $w_{2 h}=z_{h}, w_{2 h+1}=a_{h}$. Then for all $h \in \mathbf{Z}$ we have

$$
\prod_{h \neq s} \Psi\left(w_{h}, w_{s}\right)= \begin{cases}\prod_{n \neq j} \Psi\left(z_{n}, z_{j}\right) \prod_{k} \Psi\left(z_{n}, a_{k}\right), & h=2 n, \\ \prod_{j} \Psi\left(a_{n}, z_{j}\right) \prod_{n \neq k} \Psi\left(a_{n}, a_{k}\right), & h=2 n+1 .\end{cases}
$$

In either case, the product on right-hand side is bounded below by the positive constant $\varepsilon c$, which is independent of $h$. Therefore, by Theorem 3, the "union" $\left(w_{h}\right)$ of the two sequences $\left(a_{k}\right)$ and $\left(z_{n}\right)$ is interpolating. Let $B$ denote the Blaschke product relative to $\left(a_{k}\right)$. Then by Theorem 4 the Blaschke quotient $B_{\left(z_{n}\right)} / B$ is normal. Since $\left(a_{k}\right)$ is interpolating in $\Delta$, by Lemma 5 , there exists $\delta>0$ such that $\left(1-\left|a_{k}\right|^{2}\right)\left|B^{\prime}\left(a_{k}\right)\right| \geq \delta, k \in \mathbf{Z}$. Consequently,

$$
\begin{aligned}
\left(1-\left|a_{k}\right|^{2}\right) \sigma_{Q}\left(a_{k}\right) & =\frac{\left(1-\left|a_{k}\right|^{2}\right)\left|B^{* \prime}\left(a_{k}\right) B\left(a_{k}\right)-B^{*}\left(a_{k}\right) B^{\prime}\left(a_{k}\right)\right|}{\left|B\left(a_{k}\right)\right|^{2}+\left|B^{*}\left(a_{k}\right)\right|^{2}} \\
& =\frac{\left(1-\left|a_{k}\right|^{2}\right)\left|B^{\prime}\left(a_{k}\right)\right|}{\left|B^{*}\left(a_{k}\right)\right|} \geq \frac{\delta}{\left|B^{*}\left(a_{k}\right)\right|} .
\end{aligned}
$$

Since $\lim _{k \mapsto \infty}\left|B^{*}\left(a_{k}\right)\right|=0$, it follows that $\left(1-\left|a_{k}\right|^{2}\right) \sigma_{Q}\left(a_{k}\right)$ tends to $+\infty$ and the assertion follows.

\section{BIBLIOGRAPHY}

1. L. V. Ahlfors, Complex analysis, 3rd ed., McGraw-Hill, New York, 1979.

2. L. Carleson, An interpolation problem for bounded analytic functions, Amer. J. Math. 80 (1958), 921-930.

3. J. A. Cima and P. Colwell, Blaschke quotients and normality, Proc. Amer. Math. Soc. 19 (1968), 796-798.

4. J. B. Garnett, Bounded analytic functions, Academic Press, New York and London, 1981. 
5. M. H. Heins, Complex function theory, Academic Press, New York and London, 1968.

6. O. Lehto and K. I. Virtanen, Boundary behaviour and normal meromorphic functions, Acta Math. 97 (1957), 47-65.

7. F. Marty, Recherches sur la répartition des valeurs d'une fonction méromorphe, Ann. Fac. Sci. Univ. Toulouse (3) 23 (1931), 183-261.

Department of Mathematics, University of Maryland, College Park, MaryLAND 20742

Dipartimento di MATEMATICA, UNIVERSitÀ Di BARI, 70125 BARI, ITALY

Current address: Department of Mathematical Sciences, George Mason University, Fairfax, Virginia 22030 